\title{
LA PERSPECTIVA ANTROPOLÓGICA EN LA ADECUACIÓN DE LA ACTIVIDAD DE UN CENTRO DE SALUD A SU ENTORNO FÍSICO Y SOCIAL: BARRIO VIRGEN DEL REMEDIO (ALICANTE).
}

MM. RIZO BAEZA*, S. FERNÁNDEZ ARDANAZ**.

* Dep. Enfermería, UA. ** Div. Antropología, UMH.

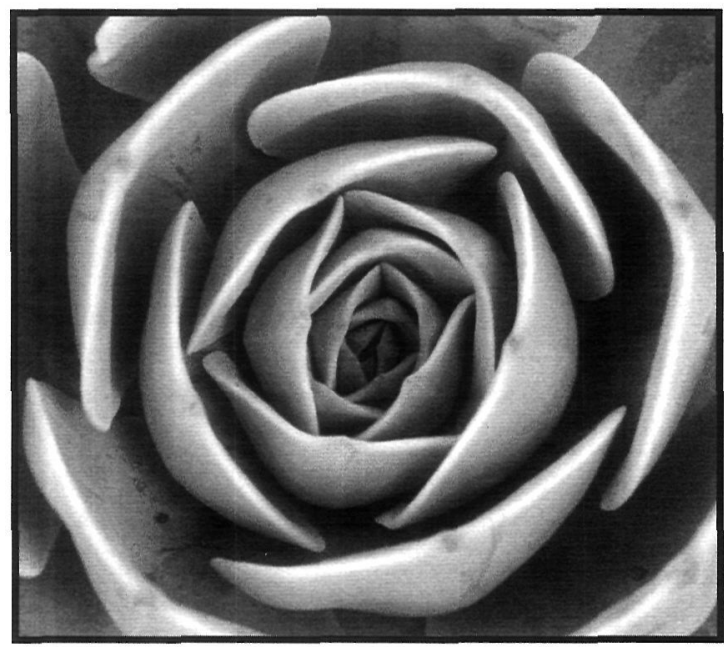

ANTHROPOLOGICAL PERSPECTIVE ON THE ADAPTATION OF THE ACTIVITIES OF A HEALTH CENTER TO ITS PHYSICAL AND SOCIAL SETTING: VIRGEN DEL REMEDIO NEIGHBOURHOOD IN ALICANTE.

$\mathrm{T}$ he relationship of a health center with its habitat as an interactive element between people and their environment is described. Within this context, the objectives are:

-Describing the habitat, the social and cultural characteristics and the relationship between people and their setting.

-Studying aspects of the built setting which can influence quality of life of its inhabitants, not merely considered as health services users.

-From this study, redefining the role of the health center as an interactive element to moderate and change that habitat, from an integral concept of health.
The data collected from the field work were used to carry out the anthropological analysis of the neighbourhood, focusing on the private space, the home, as an anthropological setting, and the public space, verifying a lack of "anthropological settings" as places where the individual global needs are met.

This neighbourhood is anthropologically a "place outside of" and "transition place", causing excision and marking the "alien" trait in front of the rest of the city, besides not creating any communication system for the population within the neighbourhood.

In this state of things, the health center must become an anthropological meeting place and a means of access to social life that not only will allow people to recover their "identity" but also will create communication nets within the neighbourhood. It is a service that "will unify everyone".

For this purpose the following needs arise:

-The staff must be permanent and has to know the anthropological characteristics of the population. The concept of family doctor and family nurse springs up again.

-Activities must be adapted to the wider aspect of the definition of health, integrating individuals and environment.

-Universality of the public health system, without extra costs or fees for the patient.

Key words: anthropological place, built setting, health center.

\section{RESUMEN}

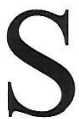
e plantea la relación de un centro de salud con su hábitat, como elemento interactivo entre habitantes y su entorno. En este contexto, los objetivos planteados son: 
- Describir el hábitat, las características socioculturales y la relación del entorno y sus habitantes.

- Estudiar los aspectos del entorno construido que pueden incidir en la calidad de vida de sus habitantes, considerados no sólo como usuarios de los servicios de salud.

- A partir de este estudio, redefinir el papel del centro de salud como elemento activo que modere y cambie dicho hábitat, desde un concepto de salud integral.

Con los datos recopilados del trabajo de campo, se hizo el análisis antropológico de un barrio, incidiendo en el espacio privado, la casa como lugar antropológico. Y el espacio público, constatándose déficit de "lugares antropológicos", entendidos como lugar donde el individuo recibe satisfacción de sus necesidades globales.

Este barrio antropológicamente es un "lugar fuera de" y "lugar de tránsito", produce exclusión y marca el carácter de "extraño" respecto al resto de la ciudad, sin crear tampoco en el interior del barrio ningún sistema de comunicación entre sus habitantes.

En estas condiciones, el centro de salud debe convertirse en un lugar antropológico, de encuentro, y de acceso a la vida social que no sólo les permita retomar "identidad" sino también crear redes de comunicación en el interior del barrio. Es "un servicio que les iguale a todos".

Para esta nueva función se hace necesario que:

- El personal de un centro de salud, sea estable y conozca las características antropológicas de su población. Resurgiendo el concepto de médico y enfermera de familia.

- Adecuar sus actividades al aspecto más amplio de la definición de estado de salud, de forma integral del individuo con su entorno.

- Universalidad del sistema público sanitario sin cuotas ni aportaciones extras económicas por uso al paciente.

Palabras clave: Lugar antropológico, Entorno construido, Centro de Salud.

\section{INTRODUCCIÓN}

En este trabajo se plantea la relación de un centro de salud con su hábitat como elemento interactivo entre los habitantes de un barrio y su entorno. De forma que se debe de establecer un flujo bidireccional entre ambos elementos, entendiendo el centro de salud no como una estructura física aislada, sino como una estructura-polo de relaciones en el ecosistema del barrio.

Para demostrar o rechazar dicha hipótesis se deberán cumplir los siguientes objetivos, que pueden expresar el esquema de actuación:

- En primer lugar, se hace necesario describir el hábitat y las características sociales, culturales, económicas, nivel de formación del colectivo humano y relaciones de los mismos con su entorno (Helman, 1994).

- Estudiar los efectos del entorno construido que pueden incidir en la calidad de vida de sus habitantes y en sus necesidades de cara al centro de salud. El marco teórico utilizado para la interpretación antropológica del espacio construido ha sido el propuesto por Rapoport (1980).

- Y con las observaciones del estudio anterior, redefinir el papel de un centro de salud teniendo en cuenta que no debe ser un elemento aislado del entorno y debe pasar a ser un elemento activo que modere y cambie dicho hábitat (Fernández, 1994).

El estudio de campo tuvo lugar entre 1990 y 1998 mediante recogida y estudio de los datos arquitectónicos, demográficos, sociales y culturales, entrevistas, observación participante y grupos de discusión. Se tuvo además en cuenta el Padrón de 1997.

La zona urbana que se describe es un barrio periurbano, situado en la zona Norte de Alicante, que aglutina desde hace treinta y cinco años a un gran porcentaje de personas que vinieron a él desde otras zonas, rurales en su mayoría, en busca de una mejor calidad de vida, siendo característico como barrio de aluvión y construido para satisfacer la gran demanda de viviendas (Mazón, 1997).

Es un barrio de Alicante con 15.156 habitantes, según el Padrón de 1997, lo que corresponde al $5,47 \%$ de la población de toda la ciudad. La mayoría de sus habitantes viven en bloques de pisos de hasta 4-5 alturas, prácticamente la totalidad sin ascensor, existiendo también un número apreciable de viviendas unifamiliares o plantas bajas, y con una media de habitantes por familia de 4-5 personas aproximadamente. 


\section{ANÁLISIS SOCIO-ANTROPOLÓGICO DEL BARRIO Y SUS HABITANTES}

Después de realizar el estudio sociológico del barrio, cuyos resultados se publicarán en otra sede, se trató de buscar las claves de su interpretación desde la perspectiva antropológica. De ahí que los aspectos que trataremos en esta breve exposición intentarán dar la visión del barrio como lugar antropológico, tanto en los espacios privados como en los espacios públicos.

Mediante estos datos y la observación de sus habitantes, costumbres, casas, modos de relacionarse etc., se puede efectuar el siguiente análisis, intentando contestar a las preguntas:

- ¿Cuáles son las características de la gente que puebla el barrio y cómo influyen en la conformación de sus entornos construidos?

- ¿Qué calidad de vida ofrece este entorno?

- ¿En que consiste la nueva función que puede tener el Centro de Salud?

- ¿Cómo y en qué medida influye el entorno físico en el comportamiento de la gente, su ánimo, bienestar, etc.?

- ¿Tiene lugares antropológicos el barrio?

A este respecto se utilizará metodológicamente el modelo del triángulo propuesto por Castells (1995), en el que el nuevo proceso urbano se crea con relación a los tres lados: tecnología, sociedad y espacio.

\subsection{El entorno físico y arquitectónico}

Es un barrio en el que sus habitantes no han construido su entorno. Fue concebido como una gran operación especulativa y saltándose la legalidad urbanística vigente (Mazón, 1994). Se realizó en una zona rural, mal comunicada y aislada del centro de la ciudad, hechos que hoy todavía son manifiestos al encontrarse como una gran bolsa en el norte de la ciudad y conectada al resto de la misma por unos pocos viales, no como continuidad de ella, como ocurre en otras ampliaciones de la ciudad, en las que el trazado de calles ha mantenido una continuidad en la expansión.

Sus habitantes, gente con modesto poder adquisitivo, compraron las viviendas ya hechas, con la promesa incumplida de la constructora de dotar con zonas deportivas, de ocio, etc (Mazón, 1994). Los jóvenes han nacido en el barrio, mientras que se alcanzan valores próximos al $90 \%$ de inmigración entre las personas mayores (figura 1).

\subsection{La casa como lugar antropológico}

Los inmuebles fueron concebidos para proporcionar casas económicamente accesibles a las personas que venían de otras zonas (rurales la mayoría) en busca de mejores condiciones de vida, pero las casas no ofrecían las mínimas condiciones de calidad en cuanto a materiales, y sin posibilidad de tener ascensores, a pesar de tener las alturas que requiere la Ley.

Físicamente todos los edificios son iguales, bloques repetitivos, de estructura monótona y encaminada al aprovechamiento máximo de la superficie edificada, casi la totalidad con 5 plantas, exceptuando mercados, iglesia y colegios.

Incluso las plantas bajas están ocupadas por viviendas, con lo que no se dejaron espacios para servicios. Las casas no ajustan el número de baños al de habitaciones y no poseen calefacción central ni aire acondicionado. Todas las viviendas poseen suministro de agua.

El espacio habitado resulta dispuesto en forma vertical y en consecuencia con muy poca libertad de movimientos y de comunicación.

El único lugar de paso a las viviendas es el portal y la escalera, la cual cumple de forma incomoda, el lugar de relación entre los vecinos. Este lugar es aprovechado por los jóvenes como lugar de relación, y por los mayores, sobre todo las mujeres, para intercambiar saludos y las preguntas cotidianas sobre el estado de salud, el tiempo y la familia.

Teniendo en cuenta que la mayoría de estas personas procedían de zonas rurales y en consecuencia de disposición horizontal, el cambio afectó profundamente a sus sistemas de comunicación, ya que en su lugar de origen éstos estaban polarizados en los lugares comunes como la calle y la plaza.

\subsection{El espacio construido público}

El espacio compartido en común por los vecinos de cada bloque es muy reducido, no permitiendo su papel de comunicación social entre ellos.

Las relaciones sociales se hacen en las plazas, sobre todo en la de Argel, que está rodeada de tiendas. No dispone de árboles ni plantas pero tiene bancos y juegos para niños. Esta plaza está estraté- 
gicamente ubicada en el centro del barrio, lugar de paso para ir al centro de salud, a los colegios, instituto, etc., siendo muy frecuentada a la hora de salida de los colegios. Hay que destacar la existencia de un quiosco de prensa en el que se sellan boletos de quinielas y lotería primitiva y que actúa como elemento de atracción, como lugar de encuentro.

Los hombres ocupan los tres bares y la bodega que allí se ubican.

\subsection{Servicios de los que dispone el barrio}

Es un barrio dormitorio, sin industrias y con pocos servicios, predominando el pequeño comercio. Posee dos mercados pequeños y dos supermercados en los que se puede adquirir cualquier tipo de productos de alimentación y de limpieza, así como tres farmacias y una óptica.

En el barrio hay contenedores de basuras y alguno de residuos específicos para reciclado que no son muy utilizados, pero existe una amplia conciencia del uso de los contenedores generales, no observándose basura fuera de los mismos, ni en horas no adecuadas.

En el aspecto de cultura, educación y sanidad, existe una biblioteca, un instituto con gran número de módulos medios y superiores, tres colegios, una guardería del Ayuntamiento y otra privada, un aula de adultos y un centro de salud.

\subsection{Espacio del ocio}

Como zona verde tiene el parque de Lo Morant situado al sur y que separa al barrio del resto de la ciudad, con abundantes zonas verdes y de ocio. En este parque, se observa que, por las mañanas y de forma casi exclusiva, caminan y toman el sol un gran número de jubilados, para los que constituye un lugar de comunicación social. Por las tardes y noches está ocupado por adolescentes y jóvenes que hacen de él su lugar de encuentro y comunicación. Es un ejemplo típico de la organización temporal del espacio apuntada por Rapoport (1980). No existe prácticamente ninguna otra zona verde.

Tiene un gran número de bares. Hay que destacar la representación del espacio que tienen estos bares, siendo lugares donde están claramente diferenciados los roles de género, puesto que están ocupados siempre exclusivamente por hombres.
También hay locales de juegos recreativos.

En contra de lo expuesto anteriormente sobre los roles de género, hay una churrería que está ocupada casi exclusivamente por mujeres. En ella se sirven desayunos hasta las doce de la mañana y el local queda cerrado hasta la mañana siguiente. Las mujeres aprovechan el momento de ir al mercado, o de la salida de gimnasia para reunirse durante unos minutos en dicho local. Es posible que se hayan creado nuevas reglas, una "antidisciplina" (Aledo, 1994), que ha permitido a las mujeres la construcción de su propio espacio social.

Existe en el barrio una organización formal exclusiva de mujeres, creada en el año 1995, "Futuro de la Mujer", con una fuerte solidaridad colectiva. Dispone de un gimnasio con monitor. Está ubicada en un chalet cedido por el Ayuntamiento. Esta organización, cuenta con más de 300 socias, y cubre unas necesidades de este colectivo, el de lugar donde reunirse, relacionarse desde el punto de vista del ocio, comunicación, de hacer deporte, hacer excursiones, etc. En resumen salir de sus labores cotidianas dentro del hogar. Necesidades que el hombre culturalmente tiene más cubiertas por sus relaciones laborales.

No existe en el barrio una distribución espacial de los locales públicos en función de la posición social de la clientela, no hay zonas de ricos y pobres como sí pueden ser encontradas en el centro de la ciudad.

No se ha observado la existencia en el barrio de ningún restaurante o local en el que se sirvan comidas.

Existe un polideportivo municipal ubicado en la zona de Ciudad Jardín, así como un gimnasio, pistas de petanca y un campo de fútbol.

\subsection{Organización de la comunicación con el resto de la ciudad}

El barrio está situado en una zona extrarradio bien delimitado y separado del resto de la ciudad a la cual se une mediante dos carreteras que lo delimitan. Existe una buena red de transportes públi$\cos$, tanto autobuses como taxis. Hay gran número de coches aparcados en la calle, al no existir aparcamientos públicos ni privados. Esta situación ha provocado la apropiación de un solar, cuyo futuro urbanístico es el de zona verde. El tipo de vehícu- 
los privados es casi exclusivamente de nivel medio, predominando los de segunda mano.

\section{ASPECTOS SOCIOLÓGICOS DE LA PO- BLACIÓN}

3.1. Características de la población según edad y sexo

Se observa en el padrón un índice de natalidad en descenso, siendo del $0,48 \%$; en 1997 sólo se constataron 100 nacimientos frente a los 14.000 aproximadamente acaecidos en la provincia de Alicante (CPS, 1997). La distribución por edades está reflejada en la figura 2 , en la que se observa que casi la mitad de la población tiene menos de 30 años, exactamente el $41,32 \%$ y que por debajo de 14 años está el $14,97 \%$ de la misma, frente al censo de 1991 en el que este porcentaje era del 30,14\%.

Al efectuar el estudio del total de hombres y mujeres se aprecia un: Índice de masculinidad, hombres/mujeres $=0,98$. Este índice es diferente al total de Alicante de 0,92 lo que indica la menor feminización de la población en el barrio, datos que no siguen la tendencia de la evolución española medida según el índice de Sundbarg (Goméz, 1995). Lo que indica una población más envejecida que la del resto de Alicante. Este hecho es un efecto de hace pocos años como se observa en la pirámide poblacional (Figura 2) y en los datos de censos anteriores.

Estos datos demográficos hacen necesario un análisis con detenimiento de lo ocurrido en el barrio, hay que tener en cuenta que en diez años se ha pasado de 24.410 habitantes a 15.156 del último censo, y que ese descenso ha llevado a un envejecimiento de la población que sigue viviendo allí.

En la pirámide poblacional se observan dos zonas disminuidas respecto a una pirámide normal, una zona alrededor de los cuarenta años y otra por debajo de los veinticinco. Este descenso no se ha producido sólo por un descenso de la natalidad, sino también por el abandono del barrio de personas de alrededor de los cuarenta, cuyo nivel adquisitivo ha aumentado y que han preferido otras zonas de mejor status social. Este hecho se constata por el número creciente de viviendas desocupadas. Estos matrimonios relativamente jóvenes se han trasladado con sus hijos adolescentes y jóvenes no casados todavía, lo que explica estas modificaciones drásticas.

\subsection{Tamaño de las familias}

El tamaño de las familias está descrito en la (figura 3 ), existiendo un $35,21 \%$ de familias que poseen 4 o más miembros frente al $34,63 \%$ en el total de Alicante. Esto está en contraposición a un hecho habitual anotado por Kottak (1997) para las sociedades industrializadas en las que está en desaparición la familia extensa.

\section{PARÁMETROS DE DEFINICIÓN SOCIO- CULTURAL}

Se ha efectuado una aproximación a la situación socioeconómica a partir de la profesión de sus habitantes (tabla 1). Predominan los trabajadores a sueldo en el sector industrial y de servicios. Hay que destacar la poca participación de la mujer en el trabajo.

Estos datos vuelven a ser un índice de la posición socioeconómica antes apuntada de tendencia hacia una posición baja y media-baja.

Los aspectos culturales también indican que el nivel de instrucción no es muy alto y apuntan también a una distribución social media-baja como se observa en la figura 4, siendo patente al comparar con los datos globales de Alicante, que el barrio está por debajo de la media, con mayores porcentajes en los niveles bajos de estudios, que se invierten en los niveles altos. Según apunta Ember (1997), conseguir más educación, en especial educación universitaria, es una de las maneras más efectivas de ascender en las sociedades contemporáneas, pero obtener educación no es nada fácil, las oportunidades de hacerlo se ven afectadas por la clase en que se nace.

Actualmente, desde las instituciones sociales del barrio (Centro de Salud, Servicios Sociales Distrito 4, colegios y el Instituto) se está potenciando el Aula de Adultos, con el fin de poder obtener una certificación de Estudios Primarios, que no poseen el $67,3 \%$ de los adultos del barrio.

Instituciones como ésta cumplen en la comunidad unas funciones de interrelación social y de mejora de la autoestima, que son observadas por los profesores como un incremento, curso a curso, del número de alumnos, sobre todo de mujeres mayores con pocas o nulas obligaciones familiares. 


\section{ANÁLISIS SOCIO-ANTROPOLÓGICO}

\subsection{La reproducción social y la producción del} espacio

El espacio estudiado es un barrio del extrarradio, marginado y periférico. Se diseñó con un claro objetivo de quedar separado de la ciudad y sin identificación con ésta, y cuya interpretación antropológica es de "lugar fuera de" y "lugar de tránsito".

Como lugar de aluvión de personas de diferentes pueblos, ha producido una exclusión y una identidad diferente al resto de la ciudad. Es un lugar construido con la finalidad de atraer a la ciudad a gente de fuera de ella, durante el momento del expansionismo urbano de los 60 , convirtiéndose en un lugar de transición, en un lugar de puro paso a la ciudad. Así, durante muchos años, el concepto de progreso social y personal de sus habitantes ha sido el éxodo, el salir fuera del barrio, e integrarse en la ciudad, siendo uno de los barrios con mayor índice de abandono de gente joven, como se observa en las diferencias entre los últimos censos.

El propio nombre, barrio Virgen del Remedio, pudo tener unas connotaciones de propaganda de venta, en el sentido de la Virgen que acoge a los foráneos y "remedia" sus problemas. La ciudad dio el nombre de su patrona al barrio, y se interpreta como un significado de bienvenida y protección a los que vienen de fuera.

\subsection{Organización del espacio}

Prácticamente la totalidad de las casas son iguales, con materiales de construcción idénticos y de baja calidad. En un estilo de construcción muy uniforme, incluso en los colores, todos iguales, de forma que nada les diferencia, más encaminado a la obtención de viviendas con poco coste y gran beneficio, más que a la comodidad de sus habitantes. Este hecho está corroborado por el proyecto original y especulativo en el que fue construido.

Sus habitantes han ido aportando elementos diferenciadores a sus casas, como acristalamiento de los balcones, toldos, ajardinado de pequeñas zonas de acera, mejoras en las porterías, pintando sus fachadas de otros colores diferentes al original, etc.

Esta forma de construcción supone una aglomeración que produce indiferenciación de identidades. Todo se homogeneiza. Sus habitantes pier- den sus costumbres y el carácter individual de su lugar de origen.

Es un barrio que tradicionalmente ha carecido de espacios sociales, hoy en día todavía no tiene cines, discotecas, ni restaurantes. No hay lugares de encuentro, siendo una de las causas de que la gente lo relacione con lugar de tránsito, que se encuentre excluida y tenga deseo de irse de él. Los jóvenes para divertirse tienen que ir al centro de la ciudad, para comer una pizza, ir a un pub o al cine.

Tiene pocos lugares de relación, y esta falta es suplida por tradicionales lugares de paso, como la plaza de Argel, que es utilizada como instrumento de comunicación tal como ocurría en sus pueblos de origen con la "plaza del pueblo", que es el lugar tradicional de comunicación personal y también tiene esa función de homogeneizar. Al igual que ocurre en los pueblos pequeños (de donde son la mayoría de los habitantes de este barrio) tiene a su alrededor locales comerciales, bares, farmacia, banco, tiendas, etc.

Tanto la tienda como los bares son los más concurridos del barrio, también son los locales más cotizados. En esta plaza además de los bares, lugar de reunión de los hombres, se encuentra la churrería, lugar de encuentro de las mujeres.

Por último, otros lugares que cumplen una función de espacio social son la iglesia parroquial, y el hogar del pensionista, pero ninguno de ellos está de alguna manera dedicado a las personas jóvenes.

En las corporaciones municipales de la democracia, se ha efectuado un esfuerzo en abandonar esta idea de barrio excluyente, mediante algunas acciones como la apertura de un gran parque colindante con el barrio, el Parque Lo Morant, que por su gran extensión toca barrios más integrados en la ciudad, pero al mismo tiempo ha servido como elemento de separación de este barrio con el resto. Hoy es un lugar donde se celebran parte de los festejos de la ciudad (Festivales de Agosto).

El Centro de Salud ha sido otro logro del barrio después de veinticinco años.

Siguiendo con esa nueva organización espacial también se están construyendo nuevos edificios en solares que estaban junto al barrio y que tienen un diseño muy diferente. En estos nuevos edificios se están utilizando colores menos uniformes, materiales de construcción mejores y lugares de encuentro 
sólo para los vecinos que allí viven, como piscinas, pistas de tenis, jardines, etc.

Estos nuevos lugares son ocupados por los habitantes de Virgen del Remedio con posibilidades económicas de cambiar de vivienda. Este hecho parece estar haciendo más patente las deficiencias de los edificios originales y del conjunto del barrio, lo que conduce a la pérdida de valor de las viviendas y su dificultad para venderlas. Puede estar detrás de este proceso otro nuevo plan especulativo que conduzca a la destrucción y nueva edificación en su lugar.

\subsection{Organización del tiempo}

Respecto a la organización del tiempo del ocio, el calendario de fiestas es idéntico al del resto de la ciudad, siendo las fiestas más aceptadas las Hogueras de San Juan. La gente mayor del barrio desde su llegada al mismo, se incorporó, con mayor ánimo incluso que los propios alicantinos a todos los rituales de esas fiestas: hogueras, barracas, desfiles, belleas del foc, etc. Esta conducta es propia de las personas que desean ser admitidas en una nueva sociedad. Pero la gente joven durante esos días, sigue bajando al centro de la ciudad, su lugar habitual de diversión.

No se han instaurado fiestas propias del barrio, lo cual propicia el efecto de ser un barrio de paso. Únicamente en los últimos años se han iniciado actividades de diversión como festivales, actividades para niños, etc. en el parque Lo Morant.

En otro aspecto, la organización del tiempo privado es la lógica de un barrio de estas características, durante el día el espacio construido es utilizado por las personas de la tercera edad, es un barrio con un alto porcentaje de clases pasivas, y por las mujeres en su paso hacia el colegio para llevar a sus hijos e ir a la compra. Los hombres y mujeres con trabajo se desplazan del barrio y los jóvenes también desaparecen al estar en colegios e instituto.

\subsection{Organización de la comunicación}

El barrio ha sido durante muchos años una isla, cumpliendo las características propias de barrio periférico. En ese sentido su papel de espacio de exclusión, al igual que los hospitales de la Edad Media fuera de las murallas de la ciudad, ha tenido su significado de espacio de tránsito, isla de aislamiento de los recién llegados hasta su tránsito a la ciudad.

Su única comunicación con el resto de la ciudad ha sido por carretera, no existiendo continuidad en el callejero hacia el resto de la ciudad. Esa marginalidad se ha intentado eliminar en los últimos años, pero quedan grandes lagunas en su integración al resto de la ciudad.

La misma construcción de los pisos colmena conduce a grandes déficits en la comunicación entre sus habitantes, siendo los únicos elementos de comunicación el rellano de la escalera y en menor medida los balcones y ventanas por la configuración propia de pisos en forma de celdillas de colmena.

\section{ACTIVIDADES DEL CENTRO DE SALUD SOBRE SU ENTORNO Y SUS PACIENTES Y PLANIFICACIÓN DE SU PAPEL FUTURO SEGÚN EL ESTUDIO ANTROPOLÓGICO REALIZADO}

El Centro de Salud se abrió en el año 1987 y no con pocas movilizaciones vecinales para que se acelerara el proceso. En el transcurso de estos trece años, el Centro y sus profesionales han ido incorporando a los habitantes del barrio una forma de comunicarse, y de acceder a la vida social diferente a la que se producía en la plaza del pueblo, iglesia, mercado, pero siendo un nuevo lugar antropológico, donde los profesionales deben de ejercer su función de guía y apoyo de todos aquellos problemas de salud que se les plantean los pacientes y que de forma directa les implica en su vida social.

Por este "nuevo" enfoque de entender la salud, creemos en la nueva "redefinición" de la función y responsabilidad del centro de salud. No se puede retrechar en actitudes que siguen generando aumento en la demanda asistencial, mayor insatisfacción por parte del usuario y aumento en el gasto farmacéutico poco sostenible. Este nuevo enfoque no se puede hacer sin contar con la participación activa de la población asignada, y el conocimiento de su habitad, de su cultura de sus costumbres y de sus expectativas en cuanto al profesional del Centro.

\section{¿Qué aporta el Centro al Barrio?}

Desde su apertura sus profesionales desarrolla- 
ron Programas de Salud, dirigidos a desarrollar la atención sanitaria en los tres niveles de salud. Dentro del programa de la mujer, se vio la necesidad que tenían de establecer programas de desarrollo ocupacional, de aprendizaje y de mantenimiento fisico para su colectivo. Para obtener ayudas institucionales se les asesoró en la creación de una asociación "Futuro de la Mujer", hasta la actualidad el grupo de mujeres es cada vez mayor y consiguen todos los años nuevas subvenciones que destinan a los proyectos que el grupo previamente establece.

Existe una solicitud intrínseca por parte de los usuarios que viene dada por la carencia actual anotada previamente, de muchos de los lugares antropológicos que estas personas han tenido en su ambiente rural previo a su inmigración.

Además de ser un lugar de encuentro, y de acceso a la vida social, el centro de salud les hace retomar "identidad", al producirse según su percepción una comunicación activa con los profesionales de la salud, que pueden acceder a un servicio médico que antes no poseían o no tenían tan fácil accesibilidad. Ésta es la diferencia, "un servicio que les iguala a todos", teniendo el mismo derecho al no depender de su nivel adquisitivo.

El centro de salud como nuevo lugar antropológico da la posibilidad de acceso a todas aquellas personas que tienen dificultades como son niños, discapacitados, ancianos, etc. El centro les ofrece un nuevo sistema de relaciones con los agentes de salud.

Frente a esta situación caben dos posibilidades: a) implicar a los profesionales en su actuación, y utilizar este gran poder para mejorar de forma eficaz los servicios que dan, e incluso utilizar su capacidad para incidir sobre otros aspectos sociales que pueden directa $o$ indirectamente mejorar la salud de su entorno; o b) introducir frenos al acceso libre y gratuito, como se propugna en ciertos grupos de gestión, mediante la introducción del "tiket moderador", que impediría a los usuarios sentirse en un lugar que les es propio.

La perspectiva antropológica que apoyamos, sobre todo en un barrio de estas características, es considerar al centro de salud como un lugar accesible a todos, que marca identidad, que hace sentirse bien al comunicarse con profesionales que saben de su vida y que les ayuda a buscar sus propios recursos, para luchar contra las adversidades del entorno.

El elemento humano que constituye el centro de salud (médicos, enfermeras y personal en general) es vínculo de identidad y de autoestima. Por lo tanto y frente a lo común en estos momentos, se hace necesaria una vinculación estable entre dicho personal y sus pacientes. Es necesaria una previsión de futuro por parte del personal y, por tanto, imprescindible su estabilidad en el trabajo. La temporalidad de este personal favorece la falta de comunicación e interacción entre paciente y personal sanitario, e impide las funciones de socialización que propugnamos.

\section{CONCLUSIONES}

- Necesidad de que el personal de un centro de salud del sistema público conozca con detalle las características antropológicas de la población sobre la que debe de actuar, así como la participación activa de la población.

- Adecuación de sus actividades al aspecto más amplio de la definición de estado de salud de su población, de forma integral del individuo con su entorno.

- Apertura de centros de salud de la red pública con personal estable. Resurgiendo el concepto de médico y enfermera de familia.

- Universalidad del sistema público sanitario sin cuotas ni aportaciones extra-económicas por uso al paciente.

\section{BIBLIOGRAFÍA}

- Aledo A (1994). Espacio y cultura. Direcciones en Sociología Urbana. Aledo A, EM Martínez y T Mazón, eds. Departamento de Ciencias Sociales. Universidad de Alicante.

- Castells M (1995). La ciudad informacional: Tecnología de la información, reestructuración económica y el proceso urbano regional. Ed. Alianza.

- Centro de Diagnóstico Precoz de Minusvalías Psíquicas. Datos año 1997. Universidad Miguel Hernández. San Juan, Alicante.

- Ember CR y Ember M. (1997). Antropología Cultural. Prentice Hall.

- Fernández S (1994). Antropología de la malattia. Serapeion (U. De la Sapienza, Roma), 115:1825. 
- Gómez R (1995). Vejez prolongada y juventud menguada. Tendencias en la evolución de la esperanza de vida de la población española, 1970-1990. Reis 71-72:79-108.

- Helman CG (1994). Culture, Health and Illness. An Introduction for Health Professionals. Butterworth Heinemann, 3 ed.

- Коттак СРн. (1997). Antropología Cultural. Espejo para la humanidad. McGraw-Hill/Interamericana de España, S.A.U.

- MAzón T (1994). La configuración del paisaje urbano en Alicante (1960-1990). Instituto de Cultura "Juan Gil-Albert". Diputación de Alicante.
- Mazón T (1997). Introducción a la planificación urbana. Ed Aguaclara, Alicante.

- Padrón de habitantes (1991). Estudios Municipales. Ayuntamiento de Alicante.

- Padrón de habitantes (1997). Estudios Municipales. Ayuntamiento de Alicante.

- RAPOPORT A (1980). Cross-cultural aspects of enviromental design. In: Human behavior and enviroment. Altman I and Rapoport A eds. NY Plenum Press.

\section{TRABAJO}

Empresarios

Pequeño comercio

Por cuenta propia

Asalariados fijos

Asalariados eventuales

Sin remuneración reglamentada

Clases pasivas
VARONES

$\begin{array}{cc}68 & 0.33 \\ 441 & 2.15 \\ 13 & 0.06 \\ 3704 & 18.06 \\ 802 & 3.91 \\ 53 & 0.25 \\ 5124 & 24.98\end{array}$

MUJERES

$\begin{array}{cc}5 & 0.02 \\ 99 & 0.48 \\ 1 & 0.01 \\ 921 & 4.49 \\ 263 & 1.28 \\ 69 & 0.33 \\ 8946 & 43.61\end{array}$

Tabla 1.

Distribución de la población del barrio Virgen del Remedio de Alicante, según trabajo ejercido.

ÍNDICE DE SUNDBARG

Población de 0 a 14 años

Población de 15 a 50 años

Población de más de 50 años
BARRIO

$15,0 \%$

$51,1 \%$

$34,0 \%$
ALICANTE TOTAL

$15,3 \%$

$53,3 \%$

$31,4 \%$.

Tabla 2.

Índice de Sundbarg de los habitantes del barrio Virgen del Remedio y comparación con la totalidad de habitantes de Alicante. 


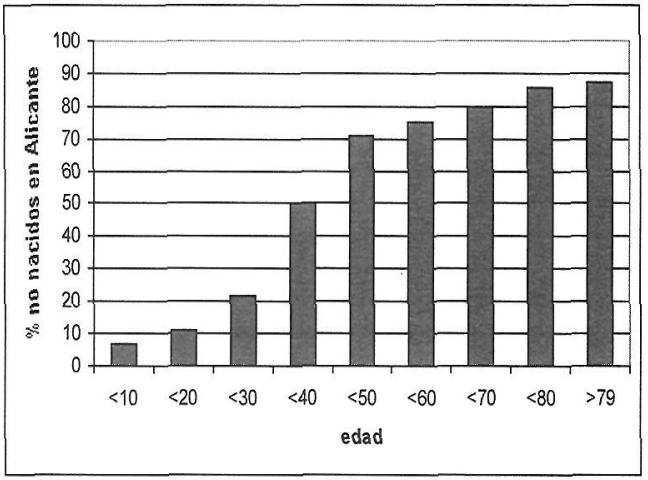

Distribución de los habitantes del barrio Virgen del Remedio de Alicante según lugar de nacimiento.

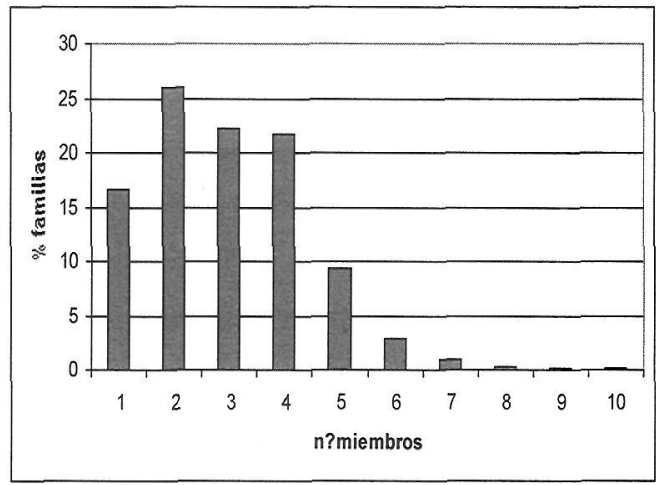

Tamaño de las familias del barrio Virgen del Remedio.

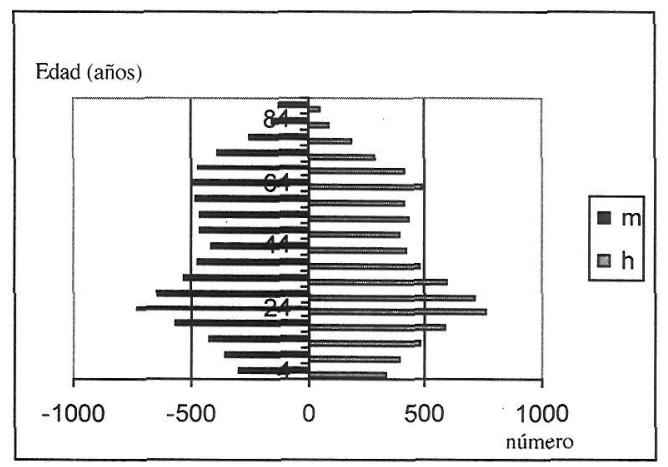

Pirámide poblacional del barrio Virgen del Remedio de Alicante según el Padrón de 1997.

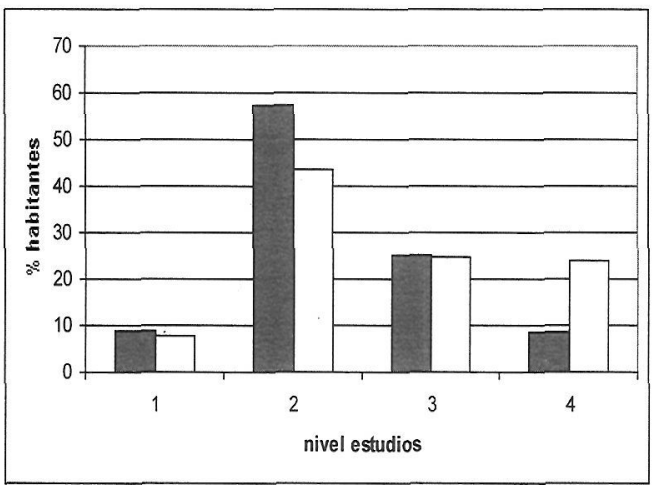

Nivel de instrucción de los habitantes del barrio. (1 no sabe leer, 2 estudios primarios, 3 estudios medios y 4 estudios superiores). 